\title{
Anabases
}

ANABASES Traditions et réceptions de l'Antiquité

$17 \mid 2013$

Varia

\section{G. KOVACS, C.W. MARSHAL, Classics and Comics}

Matthieu Soler et Mathieu Scapin

\section{OpenEdition}

Journals

Édition électronique

URL : http://journals.openedition.org/anabases/4274

DOI : 10.4000/anabases.4274

ISSN : 2256-9421

Éditeur

E.R.A.S.M.E.

Édition imprimée

Date de publication : 1 mars 2013

Pagination : 288-289

ISSN : 1774-4296

\section{Référence électronique}

Matthieu Soler et Mathieu Scapin, « G. kovacs, C.W. marshal, Classics and Comics », Anabases [En ligne], 17 | 2013, mis en ligne le 01 avril 2013, consulté le 22 septembre 2020. URL : http://

journals.openedition.org/anabases/4274 ; DOI : https://doi.org/10.4000/anabases.4274

Ce document a été généré automatiquement le 22 septembre 2020.

(c) Anabases 


\title{
G. KOVACS, C.W. MARSHAL, Classics and Comics
}

\author{
Matthieu Soler et Mathieu Scapin
}

\section{RÉFÉRENCE}

G. KOVACS, C.W. MARSHAL, Classics and Comics, Oxford-New York, Oxford University Press, 2011,265 p.

65 livres / ISBN 978-0-19-973418-4.

1 Les amateurs de comics attendaient un ouvrage de ce type, mais si le sommaire met en appétit les lecteurs, l'ensemble reste trop hétérogène. Il propose aux lecteurs de comics de comprendre comment interagissent romans graphiques et Antiquité à travers le décryptage d'exemples choisis par des chercheurs. De plus, la question se pose de la pertinence de publier ce volume aux Presses universitaires d'Oxford, alors qu'il trouverait sa place plutôt auprès des maisons d'édition de comics. Le premier chapitre est consacré à l'analyse des paramètres permettant de saisir la frontière entre les comics et l'Antiquité. Le deuxième propose des études de cas centrées sur des figures mythiques. L'étude historique fait l'objet de la troisième. Enfin, la dernière partie s'intéresse à la production d'E. Shanower, L'Âge de bronze, et à des versions européennes atypiques de l'Odyssée.

L'ouvrage s'ouvre sur une intervention de G. Kovacs visant à établir des outils critiques. La réflexion de l'article est fondée plus particulièrement sur l'étude d'Alley Oop, paru en avril 1939, qui serait le premier comics à se dérouler dans l'Antiquité. Ce sont aussi les premières planches à faire l'objet d'une réflexion sur la relation entre comics et classics. G. Kovacs propose une liste de références, dans le domaine de la réception, presque exclusivement anglo-saxonnes, sans citer les autres chercheurs européens. Il assimile le comics, après en avoir présenté l'histoire, aux arts visuels anciens. Ces derniers ne sont pas définis ; aucune réflexion sur l'artisan et l'artiste dans l'Antiquité et leur rapport au 
statut de l'auteur à l'époque contemporaine n'est esquissée, ce que l'on ne peut que regretter vu les problématiques envisagées.

3 La première partie de l'ouvrage s'intitule : «Seeing the Past through Sequential Art.» Elle réunit quatre interventions fort hétérogènes: deux interrogent des sources anciennes, une présente le manga japonais et la dernière développe l'archétype de la vie du héros. G. Nisbet mentionne le papyrus dit «d'Héraklès» (P. Oxy. XXII 2331), fragment de texte versifié en hendécasyllabe du milieu du III $^{\mathrm{e}} \mathrm{s}$. ap. J.-C. accompagné de dessins. Il tente de démontrer que les dessins illustrant les travaux d'Hercule ont une portée satirique qui entre en résonance avec le texte. La deuxième contribution a la volonté d'étudier le rapport entre comics et ekphrasis à travers l'exemple du bouclier d'Achille (Iliade XVIII, 483-608). K.P. Johnson estime que la lecture des différents éléments du bouclier d'Achille crée une impression d'action narrative. La troisième contribution apporte un éclairage stimulant sur le manga. N.A. Theisen s'intéresse au point de vue de l'auteur et des lecteurs, qui n'ont pas le même ressenti, qu'ils soient japonais ou occidentaux. Il fonde son étude sur le Chant d'Apollon d'Osamu Tezuka de 1970. Enfin, M.B. Rogers, interroge le stéréotype du héros, archétype de la liberté, qui est en lui-même un paradoxe car il semble excéder les bornes de l'imagination humaine alors qu'il est limité par le champ de possibles de la pensée.

4 La deuxième partie, "Gods and Superheroes ", se consacre à des études de cas. Elle est la plus homogène du volume, atteignant un équilibre entre l'utilisation de l'Antiquité dans les comics et l'étude sémiologique qui en ressort. C.W. Marshall étudie l'image des Furies dans de nombreux allers-retours entre la littérature classique et les créations graphiques (Wonder Woman par exemple). L'image divine n'est pas en reste lorsqu'elle est accompagnée de contextes historiques en lien avec la création du comics (Coming up to Code de Craig Dethloff, The Burden of War de R.C. Simms) ou de références bibliques (Seven Thunders utter Their Voices de Benjamin Stevens).

5 La troisième partie, "Drawing (on) history ", est moins cohérente. Les deux premières contributions s'intéressent à l'œuvre de F. Miller. V. Tomasso interroge, en ayant recours à la narratologie, la relation entre Dwight McCarty, héros de Sin City, et les Spartiates à partir d'une scène faisant allusion aux Thermopyles. Miller utilise ce passage pour marquer la différence entre modernité occidentale et vertus supposées des Anciens. Puis E. Fairey tâche de comprendre, à travers l'exemple de 300, comment, à son avis, les vases peints véhiculent des narrations et des idéologies, de la même façon que le comics. Cette analyse souffre d'un manque de rigueur méthodologique car elle compare deux contextes de pensée bien différents sans définir chacun d'eux avec précision et justesse ; les travaux d'A. Bouon et F. Lissarrague sont ignorés. D.K. Strong analyse ensuite un épisode de Sandman centré sur Auguste, ouvrage faisant partie du renouveau du comics historique, marqué par un freudisme débridé. Le dernier article, de M.T. Dinter, présente les bandes dessinées francophones. Le lecteur européen n'y trouvera rien de neuf.

6 Le dernier grand ensemble d'études de cet ouvrage favorise, par deux articles, L'Âge de Bronze d'E. Shanower. Cet auteur, qui a aussi publié un article dans The SAA Archaeological Record, propose une intervention imagée rendant compte de sa démarche de création. Cependant, ses lecteurs seront déçus de voir qu'il a "recyclé » la postface présente notamment dans l'édition française du premier tome de L'Âge du bronze. Son article dans ce présent ouvrage est intéressant, mais n'apporte aucune information supplémentaire. «Eros conquers all » de C. Sulprizio s'intéresse au rôle de l'Amour se 
substituant aux interventions divines. Elle explique que L'Âge de bronze est avant tout une main tendue aux lecteurs de comics non assidus. Si Sulprizio analyse de manière intéressante le rôle d'Eros, elle ne pousse pas assez l'étude iconographique de l'amour et de la séduction dans L'Âge de bronze, en rapport avec les conceptions que les Anciens s'en faisaient. Examiner la façon dont Shanower a saisi le rôle de l'Amour chez les populations hellènes de l'Antiquité pour les réintégrer dans sa propre vision de la guerre de Troie semblait pourtant au cœur de la démarche de ce volume. Quant à la dernière intervention, $\mathrm{T}$. Jenkins y étudie deux romans graphiques français qui mettent en scène le voyage d'Ulysse dans une ambiance érotique.

7 En définitive, l'absence d'introductions et de conclusions pour chaque section ne fait que renforcer l'impression de confusion qui règne à l'intérieur des parties. L'ouvrage est destiné avant tout à initier les lecteurs passionnés de comics plutôt qu'à faire progresser la recherche dans ce domaine.

\section{AUTEURS}

\section{MATTHIEU SOLER}

Université de Toulouse (UTM)

soler.matthieu@laposte.net

MATHIEU SCAPIN

Université de Toulouse (UTM)

mathieu.scapin@gmail.com 